\title{
Tratamientos para dejar de fumar, disponibles en México
}

\author{
Raúl H Sansores, MD, MSc, ${ }^{(1)}$ Alejandra Ramírez-Venegas, MD, MSc, ${ }^{(1)}$
}

Marlene Espinosa-Martínez, MD, ${ }^{(1)}$ Ricardo A Sandoval, Dr..$^{(1)}$

\section{Sansores RH, Ramírez-Venegas A, Espinosa-Martínez M, Sandoval RA. Tratamientos para dejar de fumar, disponibles en México.}

Salud Publica Mex 2002;44 supl 1: S116-S124. El texto completo en inglés de este artículo está disponible en: http://www.insp.mx/salud/index.html

\section{Resumen}

O bjetivo. Describir las estrategias terapéuticas disponibles para ayudar a los fumadores a dejar de fumar. Material y métodos Estudio realizado en el Instituto $\mathrm{N}$ acional de Enfermedades Respiratorias, México. Se hizo una revisión en Medline con el encabezado de meta-análisis y se consultó el Cochrane Library, de 1990 a 2001. Resultados. La farmacoterapia muestra una buena probabilidad promedio de éxito para dejar de fumar expresada como una relación entre el medicamento activo y el placebo (índice de 39, 78, 79,117 y $119 \%$ para los chicles de nicotina, los parches de nicotina, el bupropión, el inhalador de nicotina y el spray nasal de nicotina, respectivamente). El éxito de la terapia conductual puede ser buena ( $R M=3.8)$, sin embargo, se requiere más investigación. Conclusiones Se hace énfasis en la necesidad de combinar la terapia co gnitivo-conductual con el uso de fármacos, así como la combinación de éstos entre sí para incrementar las posibilidades de éxito para dejar de fumar. El texto completo en inglés de este artículo está disponible en: http://www.insp.mx/salud/index.html

Palabras clave: tabaquismo; farmacoter apia; auxiliares para dejar de fumar; México

\author{
Sansores RH, Ramírez-VenegasA, \\ Espinosa-Martínez M, Sandoval RA. \\ Smoking cessation therapies \\ available in Mexico. \\ Salud Publica Mex 2002;44 suppl 1: S116-S124. \\ The English version of this paper \\ is available at: http://www.insp.mx/salud/index.html
}

\begin{abstract}
A bstract
Objective.To describe smoking cessation therapies available in Mexico. Material and Methods Literature review of meta-analysis, controlled clinical trials, and behavioral therapy studies. Results. Smoking cessation pharmacotherapy interventions showed a good chance of success on aver age, expressed as the ratio of the active drug vs. placebo cessation therapy outcomes (ratios of 39, 78, 79, 117, and $119 \%$, for nicotine chewing gum, bupropion, nicotine patch, inhaler, and nicotine nasal spray, respectively). Behavioral therapy showed satisfactory results, $(O R=3.8)$ however, more research is needed to establish its effectiveness. Conclusions Emphasis is made on the need to combine behavioral therapy with pharmacotherapy, to increase the likelihood of successful smoking cessation. The English version of this paper is available at: http://www.insp.mx/salud/ index.html
\end{abstract}

Key words: smoking; pharmacotherapy; smoking cessation; Mexico
E 1 tabaquismo es una enfermedad y el fumador es E un enfermo. Es una enfermedad a la que es más fácil definir como un síndrome de daño multisistémico, lentamente progresivo, asociado con la adicción a la nicotina. ${ }^{1}$ El fumador es un enfermo en los términos del Manual Diagnóstico de Trastornos Mentales (DSM-IV). ${ }^{2}$ Cursa con una adicción severa que a su vez condiciona dependencia psicológica y física. Sus características son las de un adicto a la nicotina y eventualmente puede desarrollar síntomas del síndrome de abstinencia al abandonar su consumo.

Tomando en cuenta que el número de enfermos que hay en México es alto (prevalencia de fumadores de $28 \%$ en la población que tiene entre 12 y 65 años de

(1) Departamento de Investigación en Tabaquismo y EPO C. Instituto N acional de Enfermedades Respiratorias, Secretaría de Salud, México, D.F., México.

Fecha de recibido: 9 de abril de 2001 - Fecha de aprobado: 8 de noviembre de 2001 Solicitud de sobretiros: Raúl H. Sansores. Departamento de Investigación en Tabaquismo y EPO C. Instituto N acional de Enfermedades Respiratorias, SSA. Calzada de Tlalpan N 0. 4502, 14080 México, D.F.

Correo electrónico: rhsansores@aol.com 
edad), ${ }^{3}$ se debe considerar que se trata de un problema de salud pública. Además, considerando que el número de enfermedades y muertes asociadas con el consumo del tabaco es también importante, ${ }^{4}$ el problema adquiere proporciones graves. Por lo tanto, se requiere de una intervención terapéutica, aun cuando nadie, en forma específica o personal, reclame o pida tal tratamiento. De la misma forma que como en otros problemas de salud pública, como cólera, tuberculosis, dengue, SIDA, etcétera, el Estado interviene para procurar salud a la población, también debería intervenir para prevenir y tratar el fenómeno del tabaquismo en México.

A pesar de toda la complejidad que existe en el proceso de empezar a fumar, mantenerse fumando y querer dejar de fumar, prácticamente la mayoría de los fumadores quiere dejar de fumar., En México, en 1999 y 2000 se hicieron aproximadamente 50000 intentos al día para dejar de fumar. ${ }^{7}$ Sin embargo, también sabemos que solamente un porcentaje muy pequeño de todos los que intentan dejar de fumar, alrededor de $2 \mathrm{a}$ $5 \%$, consigue mantenerse en abstinencia durante un año ${ }^{8,9}$ Este tiempo de abstinencia puede prolongarse en forma variable porque desafortunadamente, aun después de varios años de abstinencia, un número importante de ex fumadores recae.

El problema es complejo. La enfermedad no es reconocida por la mayoría de la población y, a menos que el fumador ya haya desarrollado alguna de las múltiples posibilidades de daño orgánico, a los fumadores no se les reconoce como enfermos. En este trabajo analizaremos las opciones terapéuticas actualmente existentes bajo un marco de propuestas factibles para su aplicación.

\section{Material y métodos}

Este estudio se realizó en el Instituto Nacional de Enfermedades Respiratorias, México. Se hizo una revisión bibliográfica sobre los métodos auxiliares para el tratamiento de la adicción a la nicotina, actualmente disponibles. Para ello se utilizaron meta-análisis, ensayos clínicos controlados y revisiones actualizadas del Centro de Prevención y Control de Enfermedades (CDC) de los Estados Unidos de América (EUA); se hizo una revisión en Medline y se consultó el Cochrane Library, de 1990 a 2001.

Terapia de remplazo de nicotina y antidepresivos. Para la terapia de remplazo de nicotina con base en chicles o parches se hizo una revisión de meta-análisis publicados. En el caso de los medicamentos más nuevos como el bupropión y los inhaladores (nasal y bucal) de nicotina se revisaron, además, los ensayos clínicos contro- lados disponibles. Se hizo una consulta adicional de la última publicación del Cirujano General de los Estados Unidos (Surgeon General), en relación con el tratamiento de la adicción a la nicotina.

Otros métodos auxiliares para dejar de fumar, y terapia conductual. Debido a que la mayor parte de los estudios conductuales publicados no son ensayos clínicos controlados, no existen meta-análisis formales. No obstante, se hizo una revisión de las guías de práctica clínica que tienen la estructura de meta-análisis. Además, nuevamente se consultó la publicación del Cirujano General.

\section{Terapia de remplazo de nicotina}

En México, la introducción y difusión de la terapia de remplazo de nicotina (TRN) empezó a finales de la década de 1980. Diez años después se iniciaron las campañas de promoción en los medios de comunicación masiva, como la televisión. En el mercado mexicano existen tres tipos de terapia de remplazo: los chicles de polacrilex, los parches y los que administran nicotina a través de un inhalador oral. En otras partes del mundo ya está disponible también el inhalador nasal. Para la prescripción o recomendación de los sustitutos de nicotina es indispensable conocer cuánta nicotina se autoadministra el fumador cuando fuma. Cada cigarrillo contiene en promedio 6 a $11 \mathrm{mg}$ de nicotina y al fumador solamente le llegan al torrente sanguíneo 1 a $3 \mathrm{mg}$. Por lo tanto, un fumador que fuma 20 cigarros al día, en realidad absorbe entre 20 y $40 \mathrm{mg}$ de nicotina al día, la cual al disolverse en sangre da una concentración promedio de 25 a $35 \mathrm{ng} / \mathrm{ml}$ al final del día. ${ }^{10} \mathrm{Al}$ decidir darle a un fumador un remplazo de nicotina es importante considerar cuánta nicotina libera el producto que se vaya a recomendar, cuánta nicotina está acostumbrado a recibir el fumador al día y cuál es la mejor forma de administrarle un remplazo.

Goma de polacrilex (chicle de nicotina). Este producto existe en el mercado mexicano desde 1986 como fármaco que requiere prescripción médica específica para su venta. A partir de 1995 se convirtió en medicamento popular y se puede adquirir en cualquier centro comercial sin prescripción médica. En México sólo existen las tabletas de $2 \mathrm{mg}$, en tanto que en otros países también se vende la presentación de $4 \mathrm{mg}$. La absorción de la nicotina a través de las mucosas del organismo es variable y depende de varios factores. Por ejemplo, la acidez de la cavidad bucal inhibe la absorción de nicotina y por lo tanto su biodisponibilidad es pobre. Por ello, los fumadores, al fumar, hacen inhalaciones profundas para disminuir el tiempo de permanencia del humo en la boca y para conseguir una rápida 
absorción a través de los capilares pulmonares. Las tabletas de polacrilex liberan solamente $50 \%$ de su contenido en la boca ${ }^{11}$ y por ello 10 tabletas de $2 \mathrm{mg}$ sólo proporcionan $10 \mathrm{mg}$ de nicotina. Esta cantidad sería claramente insuficiente en un fumador de 20 cigarrillos al día y probablemente experimentaría grados variables de síntomas de abstinencia. Por lo tanto, se deberá recomendar el uso de un mayor número de tabletas al día para conseguir los niveles óptimos para el fumador. De hecho, la caja en la que vienen empaquetados recomienda que se utilice el número que se requiera, de acuerdo con su necesidad de fumar sin exceder de 30 piezas de las de $2 \mathrm{mg}$. Para que la absorción de la nicotina sea óptima requiere de un $\mathrm{pH}$ alcalino en la mucosa bucal; cualquier bebida ácida como el café o los refrescos conocidos como de Cola disminuyen su absorción. ${ }^{12}$ Debido a ello, es recomendable que los usuarios no coman ni beban nada (excepto agua) durante cuando menos los 30 minutos que preceden al uso del chicle.

Eficacia. Los chicles de nicotina son eficaces auxiliares en el proceso para dejar de fumar prácticamente en todas las circunstancias en las que se utilicen, ya sea que se los proporcionen sus médicos, o por autoprescripción del fumador o por alguna clínica especializada. ${ }^{13,14}$ En varios estudios se observa que su uso a dosis altas (chicles de $4 \mathrm{mg}$ ) es efectivo en fumadores con mayor dependencia a la nicotina que en aquéllos cuya dependencia es baja. ${ }^{15-18} \mathrm{Otros}^{19}$ sugieren que la eficacia de los chicles es una función directa del grado de dependencia de los fumadores. En nuestra experiencia, el apoyo psicológico es fundamental para su funcionamiento. En un análisis de cinco años consecutivos, en la Clínica para Dejar de Fumar, del Instituto Nacional de Enfermedades Respiratorias, en México, ${ }^{20}$ se encontró que en la medida en que los pacientes reciban apoyo psicológico especializado la necesidad de los chicles de nicotina es menor. Estas observaciones son consistentes con un meta-análisis que cuestiona el valor a largo plazo de los chicles en ausencia de terapia psicosocial. ${ }^{21}$ Sin embargo, uno más reciente ha sugerido que los chicles son igualmente útiles aun sin el uso de terapia conductual de apoyo. ${ }^{22}$ En el cuadro I se citan tres meta-análisis ${ }^{22-24}$ sobre la utilidad de los chicles de nicotina de $2 \mathrm{mg}$. El resumen de éstos es que el uso de los chicles brinda una posibilidad 1.6 veces mayor de éxito que el uso de placebo (Razón de momios -RM:1.6). Es decir, son útiles en el proceso para dejar de fumar y el impacto es significativamente superior al del placebo. Esta conclusión se recogió en la última edición del Cirujano General. ${ }^{25}$

Parches de nicotina. Existen en México al menos dos tipos comerciales de parches de nicotina. Uno de ellos,

\section{Cuadro I \\ RESUMEN DE META-ANÁLISIS SOBRE LA UTILIDAD DE LA TERAPIA DE REMPLAZO DE NICOTINA, 1990, 2001}

\begin{tabular}{llccc}
$\begin{array}{l}\text { Número de } \\
\text { estudios }\end{array}$ & Referencia & $\begin{array}{c}\text { Tiempo de } \\
\text { evaluación }\end{array}$ & $\begin{array}{c}\text { Razón de } \\
\text { momios }\end{array}$ & $\begin{array}{c}\text { Intervalos de } \\
\text { confianza }\end{array}$ \\
$\begin{array}{c}\text { Chicles } \\
13\end{array}$ & Fiore $^{(23)}$ & 5 meses & 1.5 & $(1.3-1.8)$ \\
\hline 42 & Silagy $^{(22)}$ & 12 meses & 1.61 & $(1.46-1.78)$ \\
\hline 108 & Silagy $^{(24)}$ & 6 meses & 1.66 & $(1.62-1.85)$
\end{tabular}

Parches

\begin{tabular}{lllrc}
11 & Po $^{(30)}$ & $3-10$ semanas & 3.10 & $(2.65-3.62)$ \\
\hline & Po $^{(30)}$ & $6-12$ meses & 2.26 & $(1.80-2.86)$ \\
\hline 6 & Gourlay $^{(31)}$ & $2-12$ meses & 3.30 & $(1.6-3.4)$ \\
\hline 9 & Silagy $^{(22)}$ & 12 meses & 2.07 & $(1.64-2.62)$ \\
\hline 17 & Fiore $^{(29)}$ & 12 meses & 3.00 & $(2.4-3.7)$ \\
\hline 108 & Silagy $^{(24)}$ & 12 meses & 1.84 & $(1.60-2.10)$
\end{tabular}

solamente está disponible al público con prescripción médica. Se presenta en parches de 10, 20 y $30 \mathrm{~cm}^{2}$, que liberan $17.5,35$ y $52.5 \mathrm{mg}$ de nicotina, respectivamente. El otro está disponible al público como producto popular y por lo tanto no requiere prescripción médica. Se presenta como parches de 21, 14 y $7 \mathrm{mg}$ cada uno. La diferencia fundamental entre estos dos parches no solamente es su disponibilidad sino también que los últimos ofrecen en forma adicional un programa de apoyo psicológico de tipo conductual y una línea telefónica de apoyo a sus usuarios durante las 24 horas del día. Los parches liberan en promedio $0.9 \mathrm{mg}$ de nicotina por hora y alcanzan las dosis sistémicas máximas en 2 a 3 días. ${ }^{25,26}$ Por lo tanto, debe tenerse en cuenta que cuando un fumador deja de fumar el mismo día que empieza a utilizar los parches, en realidad no va a alcanzar los niveles óptimos ofrecidos por los mismos hasta que hayan transcurrido cuando menos 12 horas y puede presentar grados variables de abstinencia. Aunque las casas farmacéuticas que fabrican los parches recomiendan no fumar mientras se usan, las evidencias científicas señalan que fumar mientras se usa algún sustituto de nicotina no incrementa el riesgo que existe cuando solamente se fuma. ${ }^{27,28}$

Además de la tradicional presentación que libera nicotina durante 24 horas, en México, como en otros países, también está disponible una presentación que libera nicotina durante 16 horas. Fiore y colaboradores demostraron que no hay evidencias de que una presentación sea más efectiva que otra..$^{29}$ Aunque las casas comerciales que promueven los parches de nicotina recomiendan 10 semanas de terapia, las evidencias 
sugieren que no hay ventajas en usarlos más de ocho semanas. ${ }^{29}$ Sin embargo, tampoco es recomendable usarlos menos de ese tiempo. ${ }^{25}$

Eficacia. En el cuadro I se muestra el promedio de probabilidades de éxito de los parches de nicotina comparados con el placebo, de acuerdo con un resumen

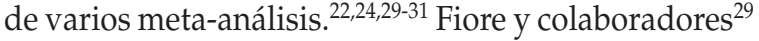
estudiaron el efecto del consejo o apoyo adicional sobre el éxito de los parches y concluyeron que el porcentaje de éxito al final del tratamiento es hasta de $42 \%$. Sin embargo, a los seis meses este porcentaje de éxito baja hasta $27 \%$. Fiore y colaboradores ${ }^{29}$ al igual que la mayoría de los meta-análisis publicados, concluyen que los parches son una ayuda efectiva en el proceso de dejar de fumar y tienen el potencial de mejorar significativamente la salud pública.

Seguridad de la TRN. Los efectos secundarios del chicle de nicotina en 3094 participantes fueron analizados en parte del Estudio de Salud Pulmonar de EUA ${ }^{32}$ y se llegó a la conclusión de que su uso no se relaciona con ninguna enfermedad cardiovascular $u$ otra enfermedad o efecto colateral serio. Benowits y Gourlay, ${ }^{28}$ en una revisión sobre los efectos cardiovasculares de la TRN, concluyeron que los riesgos asociados con ella, incluso para quienes tienen alguna enfermedad cardiovascular subyacente, son pocos y, en todo caso, siempre son menores que seguir fumando. Los efectos secundarios más importantes de los chicles están relacionados con el mal sabor o la irritación gástrica, efectos que no se observan con los parches. Con éstos, el principal problema suele ser la irritación de la piel, que se elimina cambiando los parches de posición o utilizando una crema de esteroides tópicos. En otra publicación, Benowitz $^{33}$ concluye que incluso en mujeres embarazadas que fuman, particularmente en aquellas que consumen 20 cigarros o más y a quienes las terapias conductuales no les han funcionado, la TRN se debe probar. Esta es una actitud médicamente aceptada y éticamente aceptable basada en los conceptos actuales de beneficios versus riesgos de la TRN.

Inhaladores de nicotina. Existen algunos estudios que prueban su utilidad en el proceso de dejar de fumar. ${ }^{34-37}$ En EUA su uso fue aprobado por la Food and Drug Administration (FDA) en 1997. En México, el inhalador se encuentra en el mercado desde el año 2000 y aunque no hay información local respecto de su utilidad, las evidencias internacionales sugieren que su uso asegura un buen porcentaje de éxito. Es posible que algunos fumadores lo prefieran debido a que el efecto de utilizarlo en la mano, con los mismos movimientos que se utilizan para tomar el cigarro, puede compensar el deseo de tenerlo. El cartucho de nicotina del inhalador contiene $10 \mathrm{mg}$ de nicotina y cada in- halación libera $13 \mu \mathrm{g}$, y esto representa en total $1 / 80$ de la cantidad de nicotina liberada de una inhalación de un cigarro promedio. ${ }^{34,35}$

Eficacia. El promedio de abstinencia a seis meses, de tres estudios ${ }^{35-37}$ diferentes, es de $23 \%$ versus $12 \%$ del placebo, en tanto que para 12 meses fue de 18.6 versus $7 \%$, respectivamente. La probabilidad promedio de éxito en dos meta-análisis ${ }^{24,38}$ fue de 2.3 en comparación con el placebo. Estas observaciones sugieren que el uso de los inhaladores puede ser una herramienta útil para ayudar a dejar de fumar. Sin embargo, se requiere más investigación respecto de sus efectos secundarios.

Spray nasal. Existe suficiente evidencia para recomendar estos productos como auxiliares en el proceso para dejar de fumar. El promedio de abstinentes a un año de cuatro estudios diferentes, ${ }^{39-42}$ fue de $24 \%$, en tanto que el promedio de abstinentes de los que usaron placebo fue de $12.5 \%$. En uno de los estudios ${ }^{42}$ la diferencia entre el spray activo y el placebo no fue estadísticamente significativa. Sin embargo, agrupados estos resultados en dos meta-análisis ${ }^{24,38}$ dan una probabilidad promedio de éxito muy alta (RM: 2.5).

\section{Intervención farmacológica sin nicotina}

\section{Antidepresivos}

Existe una amplia variedad de medicamentos utilizados como auxiliares para dejar de fumar. La mayoría no tienen aprobación para ser utilizados como tales, con excepción del bupropión.

Bupropión. El bupropión se comercializó durante muchos años en los EUA como un antidepresivo. En forma anecdótica se observaron sus propiedades como auxiliar en el proceso para dejar de fumar. Posteriormente, en cuando menos dos ensayos clínicos controlados bien diseñados, se probó su utilidad. ${ }^{43,44}$ Se cree que su mecanismo de acción depende del bloqueo de la recaptura de neurotransmisores en los sitios dopaminérgicos y adrenérgicos en el sistema nervioso central. Ha mostrado una utilidad dos veces mayor a la reportada para el placebo. En uno de los ensayos clínicos ${ }^{44}$ en el que se comparó bupropión/parches de nicotina/bupropión + parches de nicotina/placebo se encontró que el bupropión consigue un porcentaje de éxito de $35 \%$ a los seis meses, y de $30 \%$ a los 12 meses en comparación de 19 y 16\% del placebo con los mismos intervalos, respectivamente. Se ha reportado que el uso de bupropión se acompaña de convulsiones, cefalea e insomnio. Al parecer, el riesgo de convulsiones existe en aquellos sujetos que tienen antecedentes de ellas. Por esta razón, es preferible evitar este medi- 
camento en alcohólicos o en quienes usan inhibidores de la monoamino oxidasa. Por otro lado, la cefalea y el insomnio se podrían atenuar si el medicamento se toma a media tarde en vez de tomarlo en la noche. La probabilidad promedio de éxito (RM) reportada en el meta-análisis de Fiore $^{38}$ es de 2.1.

\section{Antihipertensivos}

Clonidina. Es un antihipertensivo que se ha utilizado tanto por administración oral como en parches transdérmicos. Aunque no ha sido aprobada por la FDA, existen varios estudios clínicos controlados que sugieren que su uso como auxiliar en el proceso para dejar de fumar puede ser muy útil. En un meta-análisis $^{45}$ se encontró que el éxito de la clonidina es mucho más alto (RM:4.2) si se agrega terapia conductual al tratamiento, que sin ella (RM:1.7). Asimismo, los parches parecen ser más efectivos que la vía oral (RM:3.2 versus 2.2). Los efectos secundarios indeseables más comunes son resequedad de boca, mareos y fatiga. Parece existir una relación causal con la dosis. Se recomienda su disminución gradual para evitar incrementos de "rebote" de la presión arterial.

\section{Resumen de eficacia de los diferentes fármacos}

En la figura 1 se muestra el efecto de los diferentes fármacos discutidos en los párrafos anteriores. La gráfica muestra la relación entre el porcentaje de abstinencia

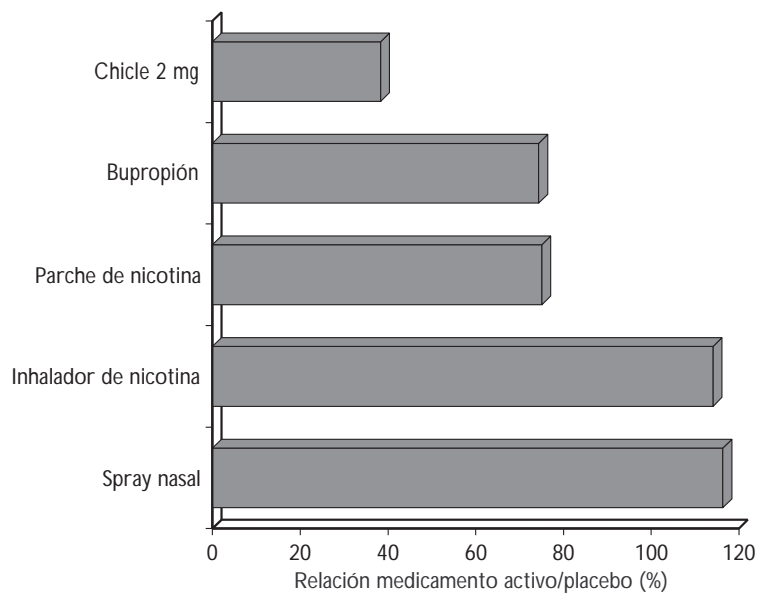

Fuente: referencia 18

Figura 1. Meta-ANÁlisis de eficaCia de Cinco terapias AUXILIARES PARA DEJAR DE FUMAR obtenido con los fármacos activos, dividida sobre el obtenido con el placebo. Se puede observar que la relación más alta se observa para el spray nasal (que no está aún disponible en México, pero ha sido aprobado por la FDA como auxiliar para dejar de fumar) y el inhalador de nicotina. Se requiere más experiencia clínica con ambos para que sus ventajas sean claramente superiores al precio económico de su uso.

\section{N uevas perspectivas farmacológicas}

El uso concomitante de dos o más modalidades de terapia no es novedoso. ${ }^{46}$ Se han sugerido nuevas opciones para el manejo farmacológico de los pacientes que quieren dejar de fumar. Existen diferentes posibilidades para asociar los parches de nicotina con los chicles de nicotina, el spray nasal, los aerosoles de nicotina y el bupropión, según propone Hughes. ${ }^{46}$ Debido a que el spray nasal no existe en México, solamente recomendamos la combinación de chicles y parches ${ }^{47}$ y la del inhalador de nicotina con parches. ${ }^{48}$ Existen algunos reportes que indican beneficios de este tipo de combinaciones. No obstante, estas aplicaciones no están aún recomendadas por ningún consenso de salud. También se ha sugerido combinar bupropión con parches como se ha hecho en estudios experimentales ${ }^{44}$ o bien, la combinación de las tres alternativas en pacientes con alta dependencia a la nicotina y trastornos de depresión mayor.

\section{Otras intervenciones útiles para dejar de fumar}

Además de la terapia farmacológica hay otro grupo de intervenciones que han funcionado con éxito relativo. En las recientes guías para el tratamiento Fiore y colaboradores $^{50}$ analizaron todas las intervenciones que han probado un éxito relativo.

Del trabajo de Fiore y colaboradores ${ }^{50}$ quedan claros algunos aspectos que enseguida resumiremos.

Toda intervención es útil para que un fumador realmente deje de fumar. Son pocas las intervenciones que reportan un éxito de cero.

Las intervenciones para dejar de fumar en las que se utilizan técnicas aversivas (como fumar rápidamente y en exceso, etcétera), incrementan la probabilidad de éxito para dejar de fumar pero deben aplicarse en personas que específicamente deseen esas técnicas, o que utilizando otras técnicas hayan fracasado.

Las intervenciones en las que hay contacto directo con los fumadores son las más efectivas. Algunas en las que no existe ningún contacto directo, parecen incrementar las probabilidades de éxito para dejar de 
fumar. De estas últimas, las líneas telefónicas que prestan ayuda y consejo a las personas que intentan dejar de fumar son más efectivas que otras estrategias.

Las técnicas que ofrecen contacto personal con los fumadores, mientras más prolongado sea el contacto mayor la probabilidad de éxito.

En el contenido de las intervenciones personales incrementa la probabilidad de éxito en la medida en que su formato incluya estrategias cognitivo conductuales como el proceso de reconocimiento de situaciones peligrosas, desarrollo de habilidades de enfrentamiento e información básica del problema del tabaquismo.

\section{Tratamiento de la dependencia psicológica}

Este aspecto no ha sido abordado con este nombre, ni siquiera por la reciente edición del Cirujano General de los EUA. ${ }^{25}$ Fiore y colaboradores lo abordan y sugieren las estrategias cognitivo conductuales que deben utilizarse. ${ }^{50} \mathrm{Si}$ bien el tratamiento de la dependencia física con alguno de los fármacos arriba descritos tiene grados variables de éxito al año, en general nunca es mayor de $40 \%$. A pesar de ello, no se ha puesto sobre la mesa el papel de la dependencia psicológica en el proceso de la recaída. Desde nuestro punto de vista, la causa final de la recaída es la dependencia psicológica. Es por ello que las terapias farmacológicas tienen poco éxito cuando se quiere evaluar su impacto un año después de haberse administrado. Es decir, el efecto de las TRN o los antidepresivos se debe evaluar a la luz de sus efectos en los primeros tres meses. Después, todo depende de las estrategias de enfrentamiento al proceso psicológico de volver a fumar. El fumador que deja de fumar nunca olvida que fue fumador y el placer que experimentó. De hecho, son todos los recuerdos de sus vivencias ${ }^{51}$ lo que probablemente desempeña un papel decisivo en la recaída. Fischer y colaboradores ${ }^{51}$ describen este proceso más detalladamente. Se basan en el placer que se experimenta con cada inhalación de un cigarro al estimular la liberación de neurotransmisores dopaminérgicos. Estas sensaciones no están aisladas de todo el contexto del conocimiento y experiencias del sistema nervioso central. Lejos de ello, se relacionan estrechamente con cada una de las neuronas corticales, de modo tal que cada experiencia de placer originada por cada inhalación de un cigarro, se asocia con una vivencia. Si a cada cigarro el fumador le hace 10 inhalaciones, entonces tendrá las correspondientes asociaciones vivenciales con las de placer. Después de 20 años de fumar 20 cigarros diariamente, el fumador ha acumulado miles de asociaciones entre cada una de las vivencias con las sensaciones de placer que la nicotina contenida en el cigarro le proporciona. Al intentar dejar de fumar, el fumador es rehén de esas asociaciones y experimenta deseos compulsivos de fumar (dependencia psicológica) cuando repite las vivencias con las que acostumbraba fumar. Por lo tanto, cualquier abordaje terapéutico estará incompleto si no se considera el aspecto psicológico de la dependencia asociada con la nicotina. La terapia cognitivo conductual aborda este tipo de dependencia y le agrega al tratamiento farmacológico un apoyo adicional para que el fumador se mantenga en abstinencia.

Terapia cognitivo conductual. El objetivo de esta terapia es cambiar un pensamiento irracional por uno racional. ${ }^{1}$ El fumador estimulado por los eventos con los que se acostumbró a asociar su conducta de fumador, reacciona irracionalmente cuando se enfrenta a ellos, aunque haya transcurrido mucho tiempo (meses o años) desde que dejó de fumar. Una variedad enorme de posibilidades pueden llevar a este cambio de conducta. En la mayoría de los métodos que son exitosos, es posible que su mecanismo de acción haya sido conductual, aunque no se promueva así. La terapia cognitivo conductual que se utiliza en el Instituto Nacional de Enfermedades Respiratorias sigue las dos estrategias generales propuestas por Fiore y colaboradores..$^{50}$ La primera estrategia general se conoce como solución de problemas/desarrollo de habilidades (cuadro II) en tanto que la segunda describe los elementos comunes de apoyo que deben utilizarse para ayudar a los fumadores a dejar de fumar (cuadro III). En el mismo documento Fiore y colaboradores aclaran que el éxito de la intervención es mayor ( $R M$ hasta de 3.8) si el equipo de salud que proporciona el servicio es multidisciplinario. Asimismo, muestra que mientras mayor el tiempo que el terapeuta invierte mayor la probabilidad de éxito. La terapia grupal parece ser más exitosa que la individual. Lo mismo ocurre con el número de sesiones. Mientras que un total de 2 a 3 sesiones proporcionan una probabilidad de éxito de $2.0(R M)$, un total de 4 a 7 sesiones incrementan el éxito (RM:2.5).

\section{Conclusiones}

El tabaquismo es un problema real de salud pública. Debido a que la adicción a la nicotina es una de las formas de farmacodependencia más difíciles de abandonar, el médico se encuentra en principio con una realidad: el fumador no quiere dejar de fumar. Se requiere por lo tanto de programas educativos y de promoción a la salud para convencerlos de que el consumo 


\section{Cuadro II \\ Estrategia 1. Elementos comunes PARA LA RESOLUCIÓN DE PROBLEMAS/DE DESARROLLO DE HABILIDADES. MÉXICO}

\begin{tabular}{|c|c|}
\hline $\begin{array}{l}\text { Componentes del tratamiento } \\
\text { para la solución de problemas }\end{array}$ & Ejemplos \\
\hline $\begin{array}{l}\text { Reconocer situaciones de riesgo } \\
\text { Identificar eventos, estados internos o } \\
\text { actividades que puedan incrementar el } \\
\text { riesgo de la recaída }\end{array}$ & $\begin{array}{l}\text { - Lo que les ocurre a otros fumadores } \\
\text { - Lo que ocurre bajo presión } \\
\text { - Ganancias del argumento } \\
\text { - Experimentar estados de urgencia } \\
\text { negativos } \\
\text { - Consumir alcohol }\end{array}$ \\
\hline $\begin{array}{l}\text { Habilidades de afrontamiento } \\
\text { Identificar y practicar el afrontamiento } \\
\text { de problemas-habilidades de solución. } \\
\text { Típicamente, estas habilidades son } \\
\text { intentos para enfrentar situaciones de } \\
\text { riesgo }\end{array}$ & $\begin{array}{l}\text { - A prender a anticiparse a las situacio- } \\
\text { nes agradables y de peligro } \\
\text { - Aprender estrategias cognitivas para } \\
\text { reducir los estados de ánimo negati- } \\
\text { vos } \\
\text { - Lograr cambios en el estilo de vida } \\
\text { para reducir el estrés, mejorar la ca- } \\
\text { lidad de vida o producir placer } \\
\text { - Aprender actividades cognitivo-con- } \\
\text { ductuales para distraer la atención de } \\
\text { la urgencia de fumar }\end{array}$ \\
\hline $\begin{array}{l}\text { Información básica } \\
\text { Proporcionar información básica sobre } \\
\text { las consecuencias de fumar y de dejar } \\
\text { de fumar }\end{array}$ & $\begin{array}{l}\text { - La naturaleza/curso de los síntomas } \\
\text { - La naturaleza de la adicción al tabaco } \\
\text { - El solo hecho de fumar (incluso una } \\
\text { sola bocanada) incrementa la po- } \\
\text { sibilidad de una completa recaída }\end{array}$ \\
\hline
\end{tabular}

de tabaco está asociado con daños a su salud, e importante morbilidad y mortalidad en nuestro país y en el mundo. Cuando el fumador contempla la posibilidad seria de dejar de fumar el sector salud debe estar preparado para ofrecerle las opciones terapéuticas más exitosas, con el fin de que el fumador lo consiga y, de ser posible, para siempre. La preparación debe orientarse en dos sentidos: a) familiarización con el consejo apropiado y b) familiarización con el tratamiento farmacológico apropiado. El consejo apropiado debe contener los aspectos que se resumen en los cuadros I, II, y III. El personal de salud que está a cargo del tratamiento y rehabilitación de los fumadores debe estar muy familiarizado con el uso de medicamentos auxiliares para dejar de fumar. En la actualidad, la combinación del tratamiento con el consejo apropiado, como la terapia cognitivo conductual y el tratamiento farmacológico disponible, incrementa significativamente la probabilidad de que un fumador deje de fumar exitosamente.

\section{Necesidades del presente y para el futuro}

Politica de salud. Aunque existen bases científicas en las cuales se funda el tratamiento de la adicción a la ni-

\section{Cuadro III \\ Estrategia 2. Elementos comunes de soporte en el tratamiento para dejar de fumar. México}

\begin{tabular}{|c|c|}
\hline Componentes de soporte del tratamiento & Ejemplos \\
\hline A nimar al paciente a dejar de fumar & $\begin{array}{l}\text { - Subrayar los efectos disponibles de ha- } \\
\text { ber dejado de fumar } \\
\text { - Resaltar a personas que hayan sido fu- } \\
\text { madores y quienes han dejado de fumar } \\
\text { - Comunicarle a los pacientes convenci- } \\
\text { dos sobre su capacidad para dejar de } \\
\text { fumar }\end{array}$ \\
\hline Comunicar y tratar & $\begin{array}{l}\text { - Preguntarles cómo se sienten al dejar } \\
\text { de fumar } \\
\text { - Expresar inmediatamente preocupación } \\
\text { y la buena voluntad de ayudar } \\
\text { - Deben expresar abiertamente sus te- } \\
\text { mores por dejar de fumar, experiencias } \\
\text { difíciles y sensaciones ambivalentes }\end{array}$ \\
\hline $\begin{array}{l}\text { Animar a que el paciente hable sobre } \\
\text { su proceso de dejar de fumar }\end{array}$ & $\begin{array}{l}\text { Preguntar sobre } \\
\text { - Razones de los pacientes para dejar de } \\
\text { fumar } \\
\text { - Dificultades para dejar de fumar } \\
\text { - Logros que se han llevado a cabo } \\
\text { - Intentos previos para dejar de fumar }\end{array}$ \\
\hline $\begin{array}{l}\text { Proporcionar información básica sobre } \\
\text { tabaquismo y el éxito de dejarlo }\end{array}$ & $\begin{array}{l}\text { - La naturaleza/curso de los síntomas } \\
\text { - La naturaleza de la adicción al tabaco } \\
\text { - El solo hecho de fumar (nivel de la } \\
\text { bocanada) incrementa la posibilidad de } \\
\text { una completa recaída }\end{array}$ \\
\hline
\end{tabular}

cotina, se requiere de una sólida voluntad política para lograr que toda la población pueda acceder a ellos. Este abordaje es, ciertamente, una forma de política de salud; debe incluir programas de promoción a la salud y de prevención de las adicciones, en particular del tabaquismo, dirigidas a los sectores joven, adolescente e infantil de la población; asimismo, favorecer en las unidades de salud de primero, segundo y tercer nivel de atención para fumadores interesados en dejar de fumar, la capacitación profesional de los trabajadores de la salud enfocados en este aspecto; por otro lado, también debe promover la investigación regional y un constante avance en materia de legislación, con el fin de proteger a los fumadores y a la población que no fuma de la exposición pasiva al humo del cigarro.

Investigación. Se requiere sin duda de investigación en materia de tratamiento. La terapia de remplazo de nicotina no estará en el mercado muchos años más con el actual estado de oferta. La nicotina es inocua cuando el tiempo y la forma de su administración se cumplen siguiendo las recomendaciones de la TRN y asegura, además, que la adicción disminuya progresivamente. Es posible, sin embargo, que se prefieran en el futuro abordajes que modifiquen en el sistema nervioso cen- 
tral los mecanismos con que funcionan los centros de "recompensa".

\section{Referencias}

1. Sansores RH , Espinosa A M. Programa C ognitivo-C onductual de la C línica para dejar de Fumar. México, D.F.: Instituto $\mathrm{N}$ acional de Enfermedades Respiratorias, 1999.

2.The American Psychiatric Association. Diagnosis and statistical manual disorders. 4th ed. DSM-IV.W ashington, D.C.:A merican Psychiatric Association, 1994.

3. Secretaría de Salud. Encuesta $N$ acional de las Adicciones. Tabaco. México 1998. México, D.F.:SSA, 2000.

4. World Health 0 rganization. Tobacco or health: A global status report. G inebra:W orld Health O rganization, 1997.

5. Fiore MC, N ovotny TE, Pierce JP, Hatziandreu Ej, Patel KM, D avis RM. Trends in cigarette smoking in the United States:The changing influence of gender and race. JAMA 1989; 261:49-55.

6. Sansores RH,Villalba-C aloca J, Herrera-Kiengelher L, Soriano-Rodríguez A, Ramírez-Venegas A. Prevalence of cigarette smoking among employees of the Mexican N ational Institute of Health. Salud Publica Mex 1999; 41:381-388.

7. Sansores RH, Giraldo-Buitrago F,Valdelamar-Vázquez F, Ramírez-Venegas A. Impacto de los medios de comunicación masiva en una campaña antitabaco. Evaluación de estrategias alternas. Salud Publica Mex 2002;xx:xx. 8.Villalba-C aloca J, Espinosa M, Sansores RH. C línicas de tabaquismo. Rev IN ER 1999; 12:83-86.

9. Fiore MC. Trends in cigarette smoking in the U nited States:The epidemiology of tobacco use. Med Clin N orth Am 1992; 76:289-303.

10. Henningfield JE. N icotine medications for smoking cessation. N Engl J Med 1995; 333:1196-1203.

11. Benowitz N L. N icotine replacement therapy: W hat has been accomplished can we do better? D rugs 1993; 45:157-170.

12. Henningfield JE, Radzius A, Cooper TM, Clayton RR. D rinking coffee and carnonatec beverages blocks absortion of nicotine from nicotine polacrilex gum. JA MA 1990; 264:1560-1564.

13. Malcolm RE, Sillett RW, Turner JA, Ball KP.The use of nicotine chewing gum as an aid to stopping smoking. Psychopharmacology 1980; 70:295-296. 14. Hjalmarson A IM. Effect of nicotine chewing gum in smoking cessation: A randomized, placebo-controlled, double-blind study. JAMA 1984; 252: 2835-2838.

15. Puska P, Björkqvist S, Koskela K. N icotine-containing chewing gum in smoking cessation:A doble blind trial with half year follow-up. Addict Behav $1979 ; 4(2) ; 141-146$

16.Tønnesen P, Fryd V, Hansen M, H elsted J, G unnersen AB, Forchammer $\mathrm{H}$ et al. Effect of nicotine chewing gum in combination with group counseling on the cessation of smoking. N Engl J Med 1988; 318(1):15-18.

17.Tønnesen P, Fryd V, Hansen M, H elsted J, G unnersen AB, Forchammer $\mathrm{H}$ et al. Two and four mg nicotine chewing gum and group counseling in smoking cessation:An open, randomized, controlled trial with a 22 month follow-up. Addict Behav 1998; 13(1):17-27.

18. Hughes JR, G ust SW, Keenan RM, Fenwick JW. Effect of dose on nicotine's reinforcing, withdrawal-suppression and self-reported effects.J Pharmacol Exp Ther 1990; 252(3):1175-1183.

19.Tang JL, Law $M, W$ ald $N$. How effective is nicotine replacement in helping people to stop smoking. BMJ 1994; 308:21-26.

20. Sansores RH, Córdoba-Ponce MP, Espinosa-Martínez M, H errera-Kiengelher L, Ramírez-Venegas A, Martínez-Rossier LA et al. Evaluación del Programa Cognitivo-Conductual para Dejar de Fumar del Instituto $\mathrm{Na}$ cional de Enfermedades Respiratorias. Rev IN ER 1998; 11:29-35.
21. Cepeda-Benito A. Meta-analytical review of the efficacy of nicotine chewing gum in smoking treatment programs.J Consul C lin Psychol 1993; 61(5):822-830.

22. Silagy $C$, Mant $D$, Fowler $G$, Lokge M. Meta-analysis on efficacy of nicotine replacement therapies in smoking cessation. Lancet 1994; 343: 139-142.

23. Fiore MC, N ovotny TE, Pierce JP, Giovino GA, Hatziandreu EJ, N ewcomb PA et al. Methods used to quit smoking in the United States: Do cessation programs help? JAMA 1990; 263:2760-2765.

24. Silagy C, Mant D, Fowler G, Lancaster T. N icotine replacement therapy for smoking cessation (Cochrane Review). Cochrane Database Sist. Rev 2001;3:CD 000146. Disponible en: http://www.update-software.com/cochrane/cochrane-frame.htm.

25. U.S. Department of Health and Human Services. Reducing tobacco U se:A Report of the Surgeon General.Atlanta (GA): U.S. D epartment of Health and Human Services, C enters for D isease Control and Prevention, $\mathrm{N}$ ational $\mathrm{C}$ enter for $\mathrm{C}$ hronic Disease Prevention and $\mathrm{Health}$ Promotion, 0 ffice on Smoking and Health, 2000.

26. Ross HD, Chan KK, Piraino AJ, John VA. Pharmacokinetics of multiple daily transdermal doses of nicotine in healthy smokers. Pharm Res 1991; 8:385-388.

27. W orking Group for the Study ofTransdermal $\mathrm{N}$ icotine in Patients with Coronary Artery D isease. $N$ icotine replacement for patients with coronary artery disease. Arch Intern Med 1994; 154(9):989-995.

28. Benowitz N L, Gourlay SG. Cardiovascular toxicity of nicotine: Implications for nicotine replacement therapy. J Am Coll Cardiol 1997; 29: 1422-1431.

29. Fiore MC, Smith SS, Jorenby JDE, Baker TB. The effectiveness of the nicotine patch for smoking cessation:A meta-analysis.JAMA 1994;271:19401947.

30. Po ALW. Transdermal nicotine in smoking cessation: A meta-analysis. Eur J Clin Pharm 1993; 45:519-528.

31. Gourlay S.The pros and cons of transdermal nicotine therapy. Med J Aust 1994; 160:152-159.

32. Murray RP, Bailey W C, D aniels K, Bjornson W M, Kurnow K, Connett JE et al. Safety of $\mathrm{N}$ icotine polacrilex gum used by 3094 participants in the lung health study. Chest 1996; 109:438-445.

33. Benowitz N L. N icotine replacement therapy during pregnancy. JAMA 1991; 266(22):3174-3177.

34. Glover ED. The nicotine vaporiser, nicotine nasal spray, combination therapy and the future of N RT:A discussion. Health Values 1993; 18:22-28. 35. Tonnesen P, N orregaard J, Mikkelsen K, Jorgensen S, N ilsson F.A double-blind trial of a nicotine inhaler for smoking cessation. JAMA 1993; 269:1268-1271.

36. Schneider N G, O Imstead R, N ilsson F, Mody FV, D oan K, Franzon M et al. Efficacy of a nicotine inhaler in smoking cessation:A double-blind, placebo-controlled trial. Addiction 1996; 91:1293-1306.

37. Hjalmarson A, N ilsson F, Sjostrom L,W iklund O. The nicotine inhaler in smoking cessation. Arch Intern Med 1997; 157:1721-1728.

38.Fiore MC, Bailey W C, C ohen SJ, D orfman SF, G oldstein MG, G ritz ER et al. Treating tobacco use and dependence. Clinical practice guideline. Rockville (MD): US. Department of Health and human services, Public Health Service, 2000.

39. Sutherland G, Stapleton JA, Russell MAH, Jarvis MJ, Hajek P, Belcher M et al. Randomised controlled trial of nasal nicotine spray in smoking cessation. Lancet 1992; 340(8815):324-329.

40. Hjalmarson A, Franzon M, W estin A,W iklund 0 . Effect of nicotine nasal spray on smoking cessation. Arch Intern Med 1994; 154:2567-2572.

41. Schneider N G, O Imstead R, Mody FV, D oan K, Franzon M, Jarvik ME et al. Efficacy of a nicotine nasal spray in smoking cessation:A placebo-controlled double-blind trial.Addiction 1995; 90:1671-1682.

42. Blöndal $T$, Franzon $M, W$ estin A. A double blind randomised trial of nicotine nasal spray as an aid in smoking cessation. Eur Respir J 1997; 10:1585-1590 
43. Hurt RD, Sachs DP, G lover ED, 0 fford KP, Johnston JA, D ale LC et al.A comparison of sustained-release bupropion and placebo for smoking cessation. N Engl J Med 1997; 337(8):595-600.

44. Jorenby DE, Leischow SJ, N ides MA, Rennard SI, Johnston JA, Hughes $A R$ et al.A controlled trial of sustained-release bupropion, a nicotine patch, or both for smoking cessation. N Engl J Med 1999; 340(9):685-691.

45. C ovey LS, G lassman AH.A meta-analysis of double-blind placebo-controlled trials of clinidine for smoking cessation. Br J Addict 1991; 86(8): 991-998.

46. Hughes JR, G oldstein MG , Hurt RD, Shiffman S. Recent advances in the pharmacotherapy of smoking. JAMA 1999; 281(1):72-76.

47. Puska P, Korhonen HJ,Vartiainen E, U rjanheimo EL,G ustavsson G ,W estin A. Combined used of nicotine patch and gum compared with gum alone in smoking cessation: A clinical trial in N orth Karelia. Tobacco Control 1995; 4:231-235.

48. Tønnesen P, Mikkelsen KL. Smoking cessation with four nicotine replacement regimes in a lung clinic. Eur Respir J 2000; 16:717-722.

49. Fisher EB, Haire-Joshu D, Morgan GD, Rehberg H, Rost K. Smoking and smoking cessation. Am Rev Respir Dis 1990; 142:702-720.

50. Fiore MC, Bailey W C, C ohen SJ. Smoking cessation. Clinical practice guideline No 18. Rockville (MD): US. Department of Health and human services, Public Health Service, Agency for health and care Policy and Research, 1996; A HCPR Publication N 0 96-0692.

51. Fisher EB, Haire-Joshu D, Morgan GD, Rehberg H, Rost K. Smoking and smoking cessation. Am Rev Respir D is 1990; 142:702-720. 